\title{
Transit GIS Applications in Fairfax County, Virginia
}

\author{
Wenyu Jia and Brendan Ford \\ Fairfax County Geographic Information Services
}

\begin{abstract}
$\overline{\text { Abstract }}$
The Fairfax County Department of Transportation (DOT) manages a fixed-route bus system (the Fairfax Connector) that encompasses 58 routes. To better support the planning, operation, and marketing of this bus system, the Fairfax County DOT and the Fairfax County Department of Information Technology formed a team to develop a pilot project of Geographic Information Systems (GIS) transit applications. These applications would serve as a demonstration to facilitate automation, analysis, accessing, and plotting of transit data. To be successful, the applications had to be cost effective and match users' technical needs with their abilities.

Paramount to the success of this project was having a transit database capable of supporting all the applications identified by the development team. The database had to handle problems such as bus stops serving more than one route and routing varying by time of day. To effectively model the bus routes in GIS, each path had to be uniquely identified even though the same route could travel on different paths. Dynamic segmentation of ARC/INFO v.7.1 was used to develop the route database. A bus stop database, in the format of ARC/INFO coverage, was then related to the route coverage.

More than 15 applications were identified for three areas of transit management: planning, operation, and marketing. Planning applications focused on transit service
\end{abstract}


improvement and route restructuring. They included routing adjustment, route demographic and land-use analysis, and reporting of statistics required by the National Transit Database. Operation applications were designed for daily service monitoring and consisted of route running times, loading at bus stops, and emergency service. Marketing applications emphasized functions for public outreach, which included creating specialized route and stop maps and publishing route information on the Internet. Most end-user applications were built in Arcliew 3.0 using Avenue and Dialog Designer. Some applications were built with ESRI's Map Objects (MO) and Map Objects Internet Map Server.

\section{Introduction}

Fairfax County is a fast-growing county in the Washington, D.C. metropolitan area. Since 1990, its population has maintained a steady growth of 2 percent from 0.8 million to 0.95 million in 1999. In the same time period, major commercial and industrial developments have occurred in the county. In the past two years, employment in Fairfax County grew at an annual pace of 5.7 percent. Growth was particularly strong in the telecommunication and information technology sectors. Growth and development coupled with aggravating traffic congestion on major corridors further pressed the need for transit service improvements.

The Transit Operations Division of the County's DOT is responsible for planning and marketing the service, and managing private operators. The Fairfax Connector carries an annual ridership of more than 4.7 million passengers. With major system expansion in July 1999, there should be significant ridership growth by the year 2000 . The issue of effective planning and efficient management, therefore, is critical.

GIS has been applied in Fairfax County's transit operations primarily for mapping needs. But it was not intended to facilitate routine tasks in a systematic approach. In May 1998, DOT introduced GIS in transit management. Input was first solicited from staff in the Transit Operations Division, and then a review was conducted between DOT staff and the County's Department of Information Technology (DIT) staff. During the process, development of a prototype for performing transit tasks through desktop GIS was desired. A list of 
selected applications was finalized for the transit GIS pilot project. In addition, a project team was formed of staff from both DOT and DIT. The team defined the pilot area: three routes and their associated stops in the southern portion of the county. These routes represent three different levels of services, including the most complex route in the system.

The objective of this project is to demonstrate the potential GIS would contribute to transit planning, marketing, and operations in Fairfax County. Therefore, it is designed to cover all three areas of interests. Planning applications address the analysis of transit service area; operation applications facilitate service monitoring; and marketing applications concentrate on public access to transit information as well as customized mapping. This article presents the results of the pilot project and discusses issues concerning systematic implementation that will follow the pilot phase.

\section{Data and Software}

The project started with the identification of existing data sources in the County in order to minimize raw data collection efforts at the initial phase. Street centerline data, already digitized by DIT, are an excellent base for route building. They offer a much higher level of accuracy than U.S. census Tiger files or any other commercially available data. They were originally produced in 1995 from 1-inch to 4,000-feet base maps. Land-use coverage data, important for planning analysis, were not readily available at this stage; therefore, a combination of the County's public facility data, planimetric data, and digital orthophotography was used to identify land-use features in the service area. Census block-group data provided a fairly detailed level of analysis. Several transit data sources are used in the applications, including transit ridership and boarding and alighting activities. The bus stop database developed by the DOT was used to create a geographic data layer of bus stops in ARC/INFO.

The digital orthophotography layer has proven extremely useful with transit applications as well as other GIS applications. The County GIS department purchased the photography in 1997 and has made it available to both County users and the public. It is viewable at an 8-foot pixel resolution on the Internet at www.co.fairfax.va.us/maps/maps.htm. The photography is served 
through a viewer (MrSid from LizardTech, Inc.). At the time of this writing, the GIS department was investigating solutions for yearly updates to this photography. Even with County personnel providing the ground control, it cost about $\$ 1,200$ per square mile. This price tag prohibits the County from updating the entire area every year. One solution is to update areas of high development each year based on available funds. Another is to update one-fourth of the county each year providing a four-year cycle of updates. Whatever the solution, the photography comes at no cost to user agencies such as transportation.

Also paramount to the success of most transit GIS projects is an up-todate street centerline layer. The street centerline layer is maintained in the GIS department and is updated as part of the GIS parcel maintenance application. Information is also provided by members of the Fire and Rescue Department and used to update the street centerline layer. As with the digital orthophotography, this information is stored on a central server and made available to all County agencies.

ARC/INFO was used to develop the route layer and the bus stop layer, both of which provide the base for the entire pilot project. Distance measures were acquired for both route and stop layers, which were later used in various applications. The development of route and stop layers is discussed in detail later in this article.

ArcView, due to its Windows-based desktop characteristics, is being used for the development of final applications for end-users.

\section{Building Routes and Stops}

As is often the case with suburban bus systems, one bus route can contain multiple paths, usually depending on the time of day, to serve varying demand. Modeling this situation in GIS requires that either each path (or route) is stored independently or that the bus route is broken down into its functional components and stored through a series of related tables. The project team contacted fellow GIS professionals with experience using the dynamic segmentation functionality in ARC/INFO. One of the most important points to consider was whether bus stops occur in the middle of street segments or at street intersections. With the former, ARC/INFO dynamic segmentation becomes much more 
a necessity. Another critical factor was the size of the route system and the degree of change. At least one site with a large route system had data stored in dynamic segmentation but was not actively maintaining the information. This site was using data from automated passenger counters to do planning and analysis tasks.

The most integrated system found with respect to both planning and operations was King County Metro in King County, Washington. This agency does not use ARC/INFO dynamic segmentation but rather a system of related tables.

A final consideration for using ARC/INFO dynamic segmentation is the change in data structures provided by ESRI. At the time of this writing, version 8 of ARC/INFO is close to final release. Part of that release features the introduction of a true object-oriented data structure. The functionality currently offered by dynamic segmentation is likely to be replaced or significantly changed in this environment.

Based on the size and small degree of change within the Fairfax system, the team decided to conduct route building in dynamic segmentation for the pilot. Based on the success of the pilot and the change in the industry, the team will reevaluate the existing dynamic segmentation functionality before final implementation.

\section{Creating Routes}

One decision that must be made when creating routes is whether to separate routes into two paths for two-directional travel. The pilot project team found it much easier to break down a bus route into one route feature for each direction and name each by direction. PACE bus service in Chicago actually includes the direction as part of the route naming convention.

Method Applied for the Pilot Project. The initial method for creating routes was to use the Select, Makeroute, Remeasure, Subselect, and Append commands in ARC/INFO to create a bus route system. While output from this method works well, it is a tedious process. This is probably the main reason that dynamic segmentation is not used in some transit agencies.

There are two other problems associated with creating routes in this fashion. First, creating routes with loops is excessively time consuming. Figure 1 
presents an example of a loop. The route must enter and leave at point A. To create the proper measurements, the user must first build the route up to point $A$ and then use the Append and Remeasure commands for further segments of the route. This is where the project team made most of its errors in building routes. A second problem occurred when the team selected arcs to include in a particular route (see Figure 2). In the selection process it appears as though there is only one arc between points A and B. The enlargement of that area, however, shows there are really two arcs. Failing to select the smaller arc will ruin the route measurements, especially when running the Remeasure command in the loop-building process. The simple solution to avoid this is to make sure node features are turned on, but this will slow down the Redraw process when panning across the network.

Preferred Method Learned from the Process. At the end of the project, the team found that ARC/INFO's Path command provides a faster way to create transit routes than the method previously described. The Path command uses either a designated file of node locations or a user-selected set of nodes to generate route features. The Path command uses a "shortest-path" algorithm to compute a route between selected nodes. This command eliminates the problems encountered in creating routes by arc selection. Because Path uses a
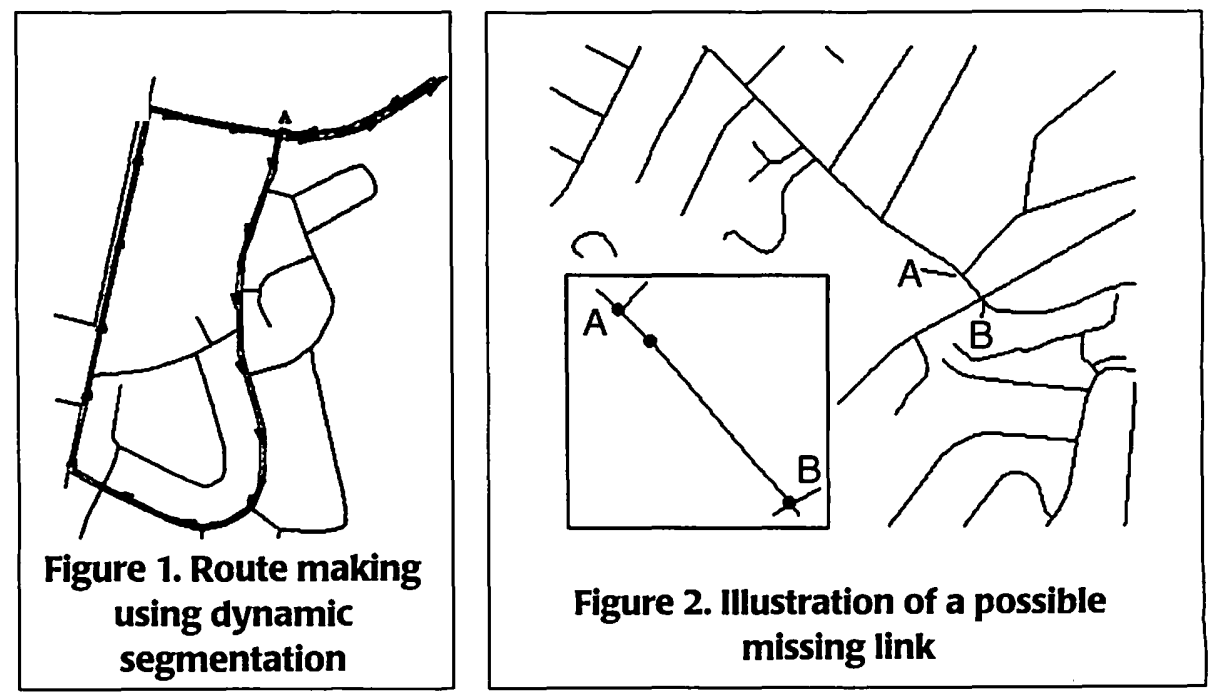

Vol. 2, No. 4, 1999 
shortest-path algorithm, there will not be any gaps in the route created. Loops are also taken care of since the stop file will be visited in sequential order. Another advantage of the Path command is that nodes used for a particular route can be saved and edited based on changes to a particular route. Recreating or altering routes becomes quite easy under this approach.

During the process, the project team also examined the possibilities of using Arc/View to create routes and stops. With the Network Analyst extension to ArcView, the user can quickly create route features using the shortestpath algorithm. Although some effort is required to link the output to the stop locations, it is an excellent and quick way to create route features. Because routes created by Network Analyst are shape files and not true ARC/INFO route systems, certain information necessary to develop comprehensive applications is missing. The project team feels the main use of ArcView and Network Analyst would be in quickly creating proposed routes for use in project analysis or for mapping purposes, while ARC/INFO would fit the best for route system maintenance.

\section{Creating Bus Stops}

Bus stop information currently exists in a paradox database. The stop location is represented by intersection attributes with "nearside" and "farside" as indications for stop distance from the intersection. The intersection attributes consisted of two fields: on-street and at-street. The results from geocoding by intersection are that the stop features are located in the middle of an intersection. Some stops are actually located 200 to 300 yards from the nearest intersection, meaning that geocoding by intersection could be misleading as to where the actual stop is located. Furthermore, during the geocoding process, a number of stops were not geocoded because, instead of containing street names, they described significant buildings (e.g., on-street: Candlewood Drive, at-street: in front of power plant).

The team evaluated the approach using a Global Positioning Systems (GPS) receiver for stop location data collection on two routes in the pilot area. With the use of a handheld GPS receiver, the team recorded accurate stop location data. The GPS receiver also allowed the team to collect field data other 
than locations. Figure 3 presents the results of the GPS data collection process. The orthophoto in the background shows the actual location of the stops collected with GPS versus the same stops geocoded by intersection. The stars in the upper-left corner of the image represent two stops geocoded by intersection attributes. The flags in the center of the image represent the actual locations of the two stops collected with GPS receivers. The flags in the lower-right corner are stops collected with GPS receivers that were not in the original stop database.

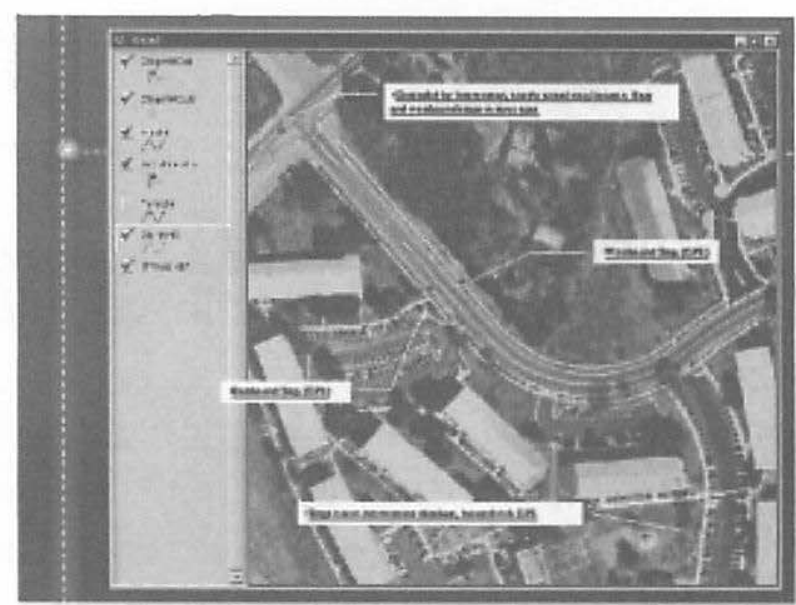

Figure 3. Comparison of stops collected by intersections and by GPS

\section{Relating Stops to Routes}

Once stop and route data are ready, they are related in ARC/INFO by the Addroutemeasure command. The only drawback to this method is that stops are related to every route within a user-specified search tolerance. For example, although five routes may pass a particular stop feature, in reality it might only be a stop for three of the five routes. It is necessary to "clean up" these extra information records. As the process is not done automatically by ARC/INFO commands, the team recommends some automation be done to carry out the task for the systemwide implementation. 


\section{Planning Applications}

Transit planning requires good knowledge of the transit services provided and the areas served. Thus, field trips have become an integral part of a transit planner's job. However, this approach in itself does not fully assist planners in acquiring an in-depth knowledge of the social, economic, land-use, and transportation features of the service area. Furthermore, field trips cannot provide a systematic ground for service justification because they are constrained by visual examination.

Desktop GIS applications can utilize and integrate data sources in the analysis and planning of transit service. Designing an automated process in ArcView could further facilitate planning analysis by supplying powerful, user-friendly tools and providing in-depth knowledge of the service area. Over the years, many transit agencies have used ArcView to develop a profile analysis of service areas. The San Diego Association of Governments developed a social-economic profile application by generating a profile of an area surrounding a transit route. They use buttons to prompt users with route number and buffer distance and develop automated spreadsheet reading of profile data.

The planning application developed by the Fairfax County DOT is unique. It creates an integrated planning process, provides planners with userfriendly tools with minimum GIS training required, and enhances the analytical function by combining decision-making processes in the design. In addition to achieving a better understanding of existing services, the application also contributes to the design of new services by assisting planners in making decisions on optimal service. The following sections describe the structure and function of the planning applications. Figure 4 illustrates the process of the planning application.

\section{Structure of Applications}

The planning application is structured into a five-step model with steps grouped under the Planning Analysis menu, as shown in Figure 5. Under the Planning Analysis menu, there are five submenus: Build New Route, Create Shed, Clip Shed, Update Info, and Export Data. Users can go through the steps in sequence depending on the type of analysis required. 


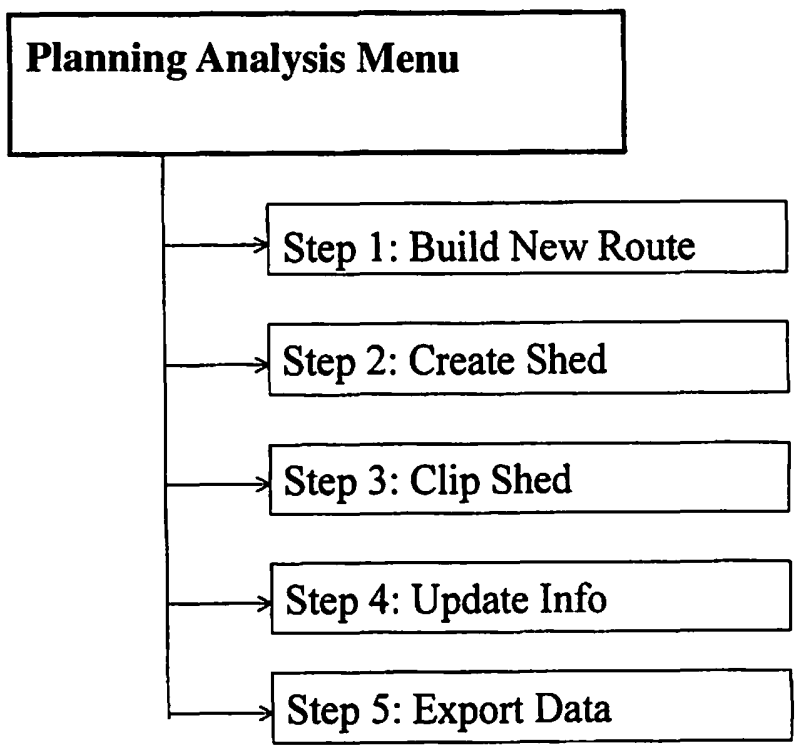

Figure 4. Planning analysis menu

\section{Analysis of Existing Service}

This analysis requires deploying only steps $2,3,4$, and 5 under the Planning Analysis menu. First, planners need to specify the shed area of the service they are studying. Starting with step 2 , they can create a shed around routes or stops within their specified buffer distance (Figure 6).

Second, planners need to find out all the transit-service-related information contained within the shed area. Using step 3, planners can clip any themes within the shed generated, including census, public facilities, and planimetric layers. However, the clipped themes contain the original attribute data in spite of the change to the original unit size. For example, in the case of census block group data, buffer areas may cover only a small portion of a block group and the clipped data actually present data for a much larger area beyond the shed. Step 4 thus functions to update data with its clipped size. By using Update Info in step 4, all relevant information will be updated based on the percentage of the original area occupied by the clipped area. The arithmetic is based on the 


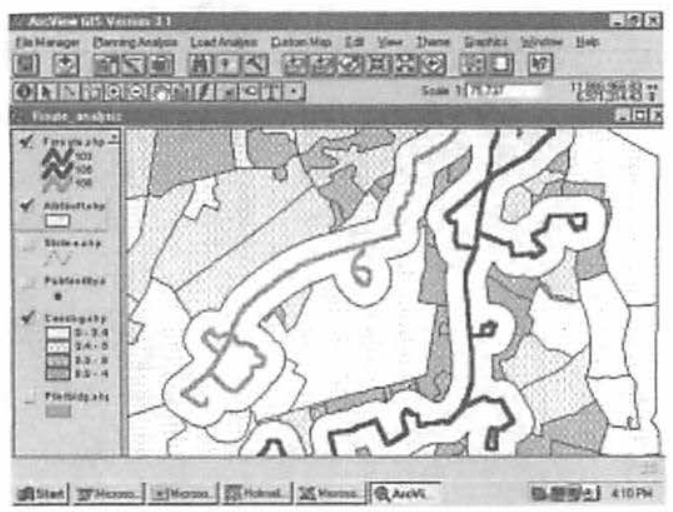

Figure 5. Menu for planning analysis

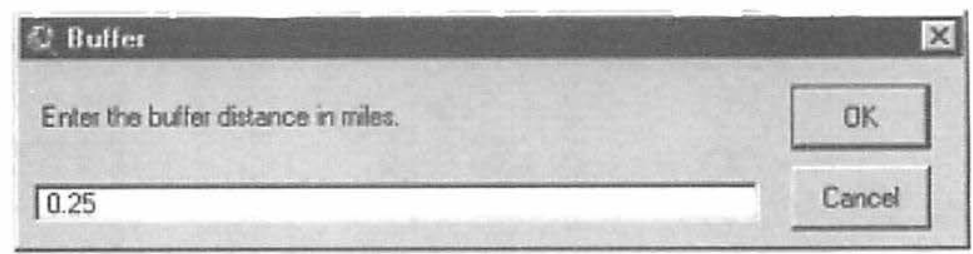

Figure 6. Dialog for creating shed

assumption that events are evenly distributed within the original spatial unit, and the Update operation should be applied only for "count" data and should not be used for density and intensity attributes. Once the data are updated, a dialog will indicate that "the table has already been updated" whenever step 4 is repeated. At this point, the user also has the option to view digital orthophotography as a means of visually verifying computed results. The unique feature of updating data with the portion of clipped area over its original significantly improves the relevance of analysis. By exporting the data in step 5, users can conduct any further (nonspatial) analysis in spreadsheet or database software.

This set of planning tools also enables planners to compare performance among routes and further analyze causes of the difference. Figure 7 presents a summary for three routes serving the Route 1 vicinity. These routes represent three different levels of performance, with Route 105 the most, Route 103 the 
moderate, and Route 108 the least productive, according to exported data from step 5 of the planning analysis. A detailed examination reveals that economic and land-use features are the underlying reasons for transit productivity along the Route 1 corridor. Route 105 serves a dense residential neighborhood with more multiple-family housing and relatively low-income level. In contrast, Route 108 is in a low-density, single-family neighborhood with the highest median household income of the three routes. Comparison of these two routes indicates that service should be enhanced in the Route 105 service area.

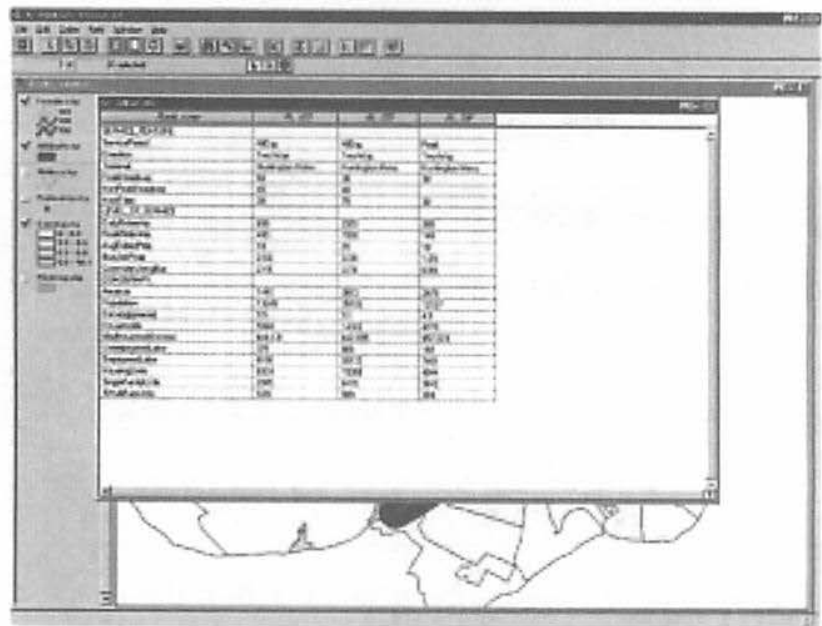

Figure 7. Summary of three routes serving the Route 1 vicinity

Furthermore, each route's information could be embedded in the application. By using hotlinks in ArcView, users can quickly find out the details of each route (Figure 8).

\section{New Route Design and Route Adjustment}

As Fairfax Connector continues to grow, new service expansion for the underserved area and adjustment of existing routes often require rigorous studies. This planning application enables planners with analytical tools to conduct scenario analysis and support decision-making in bus service design. 


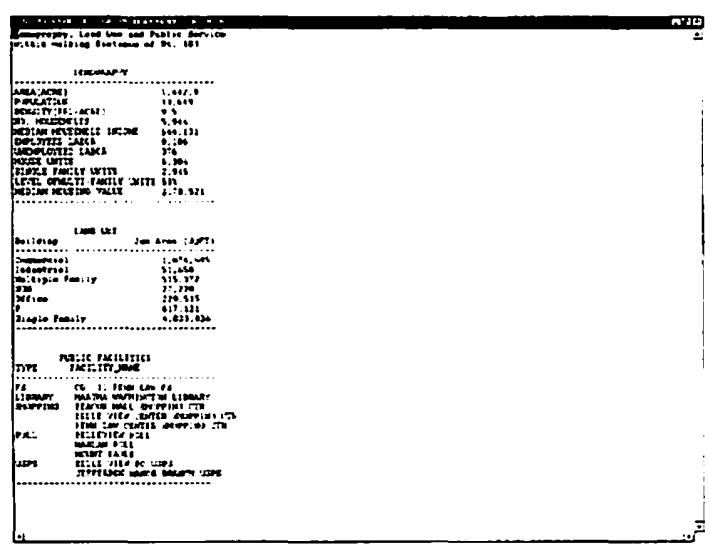

Figure 8. Summary of route information

To design the new route, planners start with step 1 under the Planning Analysis menu. The new route should follow streets on which it operates, but it does not need to be precisely overlaid above streets. After inputting the new route, planners can continue through steps 2 through 4 and quickly generate a summary of social, economic, demographic, and public service characteristics. By deploying different scenarios of new routing plans and developing service profiles for each, planners can easily compare the advantages and disadvantages of each design and select the most desirable service.

Overall, this planning application, designed in a user-friendly desktop environment, extends planners' capacity in either daily routine or major service planning. In addition to obtaining a profile of baseline features of the service, scenario analysis will play a critical role in the planning decision-making. While field observations are always essential to transit planning, the substantial amount of data presented by planning tools will further enhance the quality of transit planning. Furthermore, no additional experience is required for GIS users to carry out the five-step planning analysis, and this could encourage the acceptance of GIS in the office. Especially for medium- and large-sized transit agencies, this provides the base for successful deployment of GIS applications in day-to-day transit planning tasks. 


\section{Marketing Applications}

Several applications were identified for marketing of the Fairfax Connector system, including an Internet route query tool. As mentioned earlier, there are three other bus systems plus a portion of the Metro subway system within the study area. A true trip-planning software would ideally encompass all of these transit systems. Currently, contractors under DOT handle all passenger information inquiries and are paid by the number of calls received. A significant number of incoming calls are simple data requests such as: "What bus route passes by my house?" With a simple Internet data viewing application, the DOT could reduce the number of calls to the operations center and, in doing so, directly cut costs.

Other transit agencies have developed more comprehensive trip-planning applications, including Oregon's Tri-Met (www.trimet.org) and California's Orange County Transit Authority (OCTA) (www.octa.net). Tri-Met has several trip-planning applications. Its call-in service gives the public a complete trip plan based on an origin and destination. This trip plan is derived from the organization's GIS data. Tri-Met also provides a trip-planning component that allows the public to download route information from the Internet and perform their own trip planning. Another trip-planning application is designed for paratransit services to support citizens with disabilities. On its Internet site, OCTA provides static route maps as well as a link to the Southern California Regional Council of Governments Transtar trip planner. This software allows citizens to perform sophisticated trip planning over the Internet based on a number of user-entered parameters.

The Fairfax team developed an application using $\mathrm{MO}$ and Visual Basic to perform route information queries. MO was chosen because it could be tested in-house and converted to a web application using Map Objects Internet Map Server, Java, and HTML. The process consists of address geocoding, specifying walking distance to the bus route, and route searching. Figure 9 shows the interface for the route query application in the pilot project.

The basic premise is that users should be able to geocode their addresses and find transit information near their locations. This application takes a user- 
entered address; identifies the location with a red dot; and then displays street, bus route, and bus stop data layers. After the user clicks the Find or Find Route button, a message box pops up showing bus routes within a user-specified distance. Once a format has been decided on, the user will then have the opportunity to view route schedules and other information. The user-entered distance is a straight buffer distance. In the future, the team may add the ability to find the closest routes by network distance. Several major transit agencies, including OCTA, define stop accessibility based on network distance instead of straight Euclidean distance.

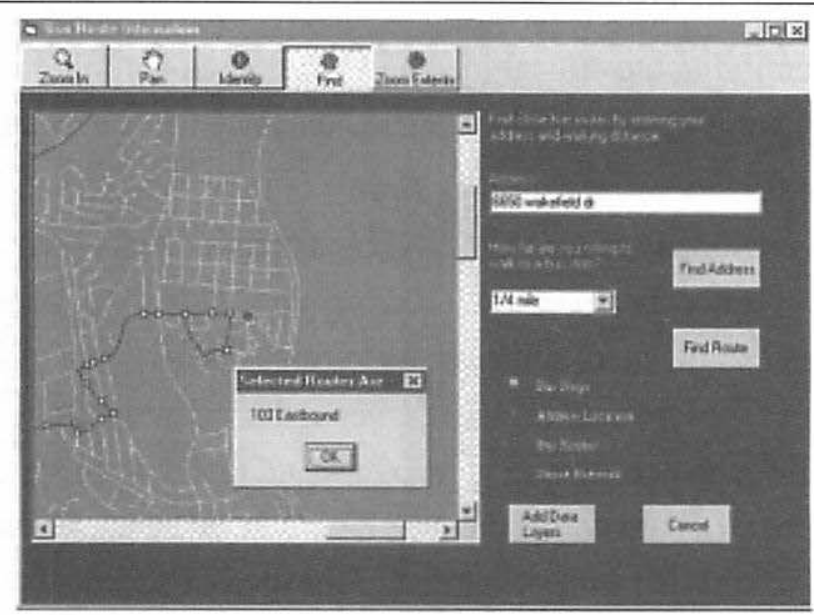

Figure 9. Illustration of route finding

Generating maps for presentations and public displays is another application identified by the marketing group. Until recently, presentation maps were done manually. Even with desktop GIS capabilities, making maps of proposed and existing routes and facilities can be time consuming. When the data conversion effort is complete, this will no longer be such a difficult task.

The marketing group also needs to display bus stops with associated attributes (e.g., shelters, benches, sign type, and whether or not a stop is a snow emergency stop). Some of this information exists and will be linked to the GPS collected stops. Information not currently available will be captured during the systemwide data collection phase. 


\section{Operation Applications}

Operation applications are designed for service monitoring and statistics. As an initial effort in application development, the project team concentrated on two areas: boarding and alighting, and route distance and running time.

\section{Analysis of Boarding and Alighting Activities}

The application provides tools for querying boarding and alighting activities by route. As shown in Figure 10, by pulling down the Operation Analysis menu and clicking on Heavy Boarding, the application will quickly query the stops and generate a table with heavy boarding activities and vice versa for the light boarding and alighting activities. This function would assist operators and planners in identifying the critical loading segments on the route and incorporate this information in service planning and enhancements.

\section{Finding Route Distance and Running Time}

Routes developed using ARC/INFO dynamic segmentation contain detailed distance information by each routing plan. This set of operation tools offers operators and planners quick access to route distance and estimated running time. Under the Operation Analysis menu, the Route Measure option will lead planners through pop-up query dialogues and report the distance measure and running time in a summary table (see Figure 11). The application for transit operations should be further explored in the area of schedule development and route statistics. More specifically, function of route statistics should be integrated later in the report to the National Transit Database.

\section{Conclusions}

The experience gained and lessons learned in the Fairfax pilot system reported here provide valuable insights in designing systemwide implementation and are instrumental in deciding the future direction of the project.

\section{Baseline Database Development}

The base required for the Fairfax project includes route and stop database and coverage. Although the team started with manual dynamic segmentation after trying several other approaches, at the end they found the Path command in ARC/INFO a most effective approach in building bus routes. It will ulti- 


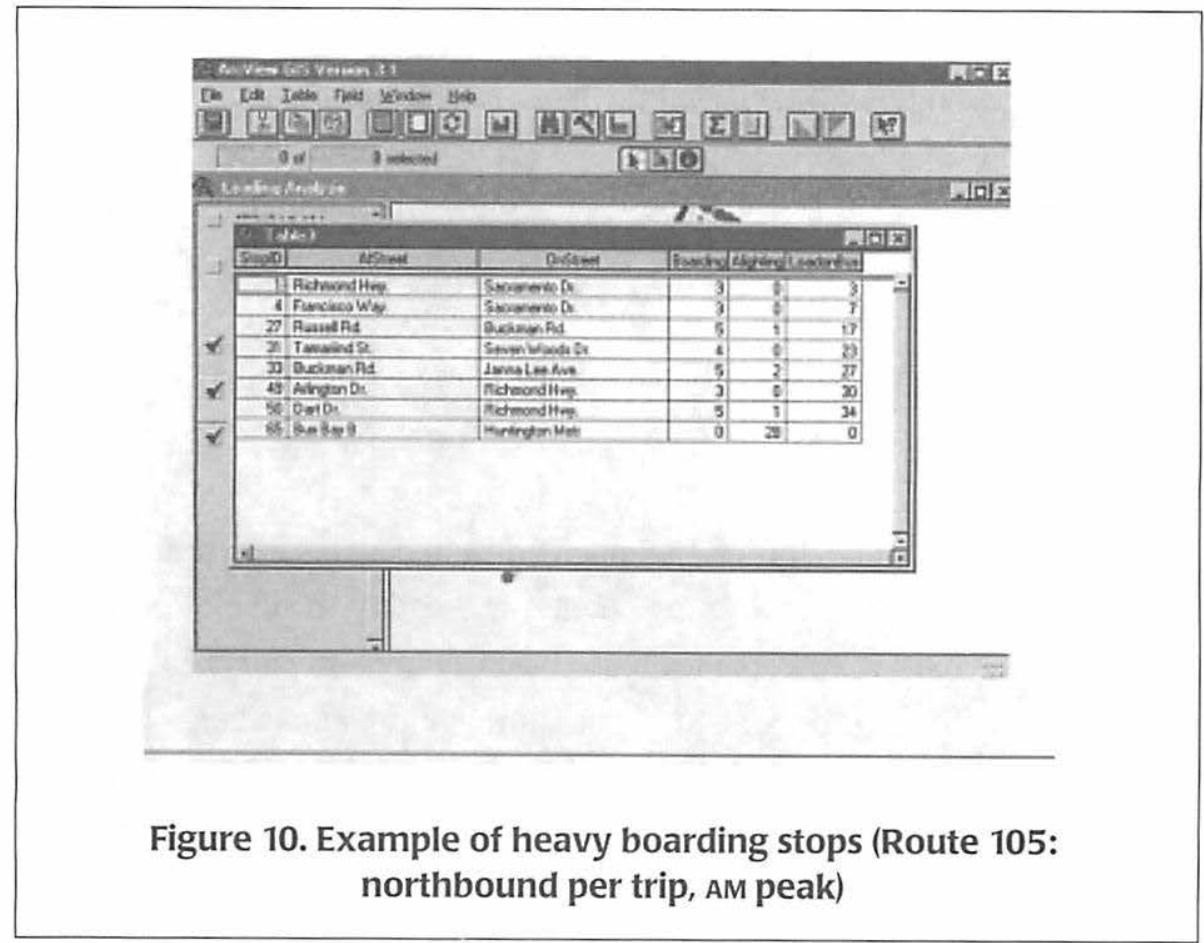

mately facilitate the maintenance and updating of bus route systems by DOT staff with some training and assistance from the GIS staff.

The existing stop data using intersection as a location indication does not reach the required accuracy. GPS equipment should overhaul the current stops

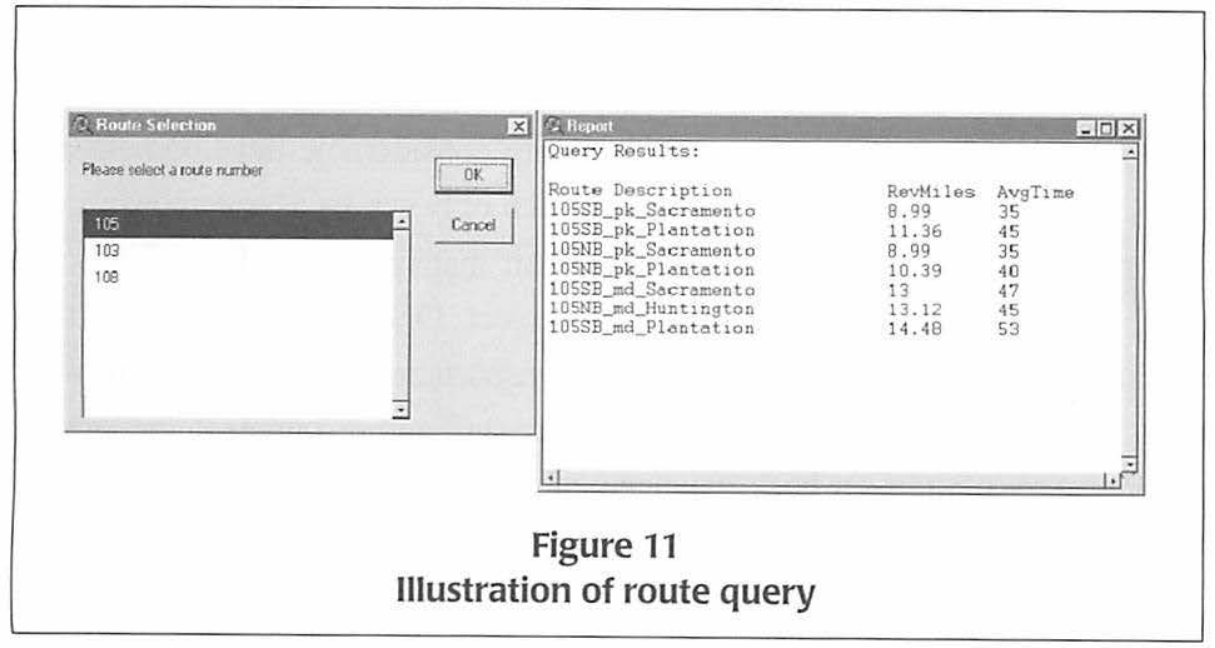


database in the systemwide implementation of the project. Existing information will be linked to GPS collected points. There is an ongoing effort by the Transit Standards Consortium to compile a set of recommended practices for bus stop data collection (www.tsconsortium.org), which could be used as a guide for future stop database collection. Transportation professionals have participated in teleconferences to identify methodologies, attribute items for collection, known problems, and many other issues associated with bus stop data collection.

\section{Data Consistency}

The Fairfax County DIT has put much effort in data collection and development. The data compiled by the County are readily available to various agencies. In the Fairfax case, the team used the County's street centerline coverage, planimetric data, orthophoto layer, and public facility data. However, they were not able to use the County's census data because the County developed it as a subcensus tract, which is different from a standard census tract. As a result, the various census data could not be used on the County-defined subcensus tracts. A decision was made at the beginning to use census block-group data, which were downloaded from the Census Bureau. Also, for the transit data, the team used data from different collection sources and no continuity could be guaranteed for the future. As the project goes into system implementation, future maintenance and update require consistency and continuity of all the data applied.

\section{Funding and Resources}

A system implementation would require considerable development and refinement of the existing pilot project, which only serves as a demonstration in terms of the role of GIS in routine transit management. Thus, resources, staff, and time would be needed for the project. One large constraint is that funding is not readily available to local transportation agencies dealing with daily system management. The need for more staff and resources in the face of funding issues could set back management incentives for systemwide implementation. 


\section{Training and Software}

Fairfax County DIT periodically offers basic GIS training to County employees. As the applications designed target minimum user experience, the training provided should be sufficient for each section in the transit operations division to leverage the capabilities of GIS technology. However, one current problem is that the DOT only has a couple of licenses for ArcView. One possible solution considered by the GIS department is to offer ArcView via a CITRIX Metaframe application. This would allow users at remote locations to access ArcView on a central server. The County currently has 80 licenses of ArcView deployed throughout the region. Serving these licenses through Metaframe would allow the County to reach more end-users. With this option the GIS department would be able to better serve transportation professionals interested in applying the planning, operations, and marketing applications.

Overall, the applications designed for day-to-day planning, marketing, and operations in the Fairfax DOT set a prototype of GIS in transit management with its unique desktop features. While the applications demonstrate potentials of GIS in transit, they will be further refined for the final system implementation based on staff comments. Once all the routes are developed, it would be just a plug-in to link the already designed application interface with the route and stop data.

\section{About the Authors}

WENYU JIA, (wjiaØl@co.fairfax.va.us) a transportation planner with the Fairfax County Department of Transportation, specializes in transit planning and operations. She holds a Master of Science in transportation engineering and a Master's degree in city planning from the University of California at Berkeley. Ms. Jia has been with the Fairfax County Transportation Department for almost two years.

BRENDAN FORD (Brendan.Ford@co.fairfax.va.us) is the team leader for the Application Development section of the Fairfax County Department of Geographic Information Services. He graduated in 1987 from Virginia Polytechnic Institute and State University with a bachelor's degree in geography and in history. He has been employed by Fairfax County for 11 years. 